\title{
PROTOCOLOS DE AVALIAÇÃO DA DEGLUTIÇÃO: NORTEADORES E LIMITAÇÕES
}

\section{SWALLOWING ASSESSMENT PROTOCOLS: GUIDELINES AND LIMITATIONS}

\author{
Jordana Balbinot ${ }^{1}$, Gabriela de Castro Machado1, Luise Stumpf Hübner ${ }^{1}$, \\ Caroline Santana Real ${ }^{1}$, Alana Verza Signorini' ${ }^{2}$, Sílvia Dornelles ${ }^{2,3}$
}

\section{RESUMO}

Introdução: A disfagia é um sintoma de alteração no processo da deglutição, que acarreta dificuldade em formar ou mover o bolo alimentar de forma segura e eficiente. Os métodos investigativos da disfagia se dividem em avaliação clínica e instrumental, os quais necessitam de protocolos que conduzam suas práticas. O objetivo desse estudo foi elencar informações acerca dos protocolos utilizados pelos fonoaudiólogos responsáveis pelas avaliações da deglutição em adultos e idosos de um hospital público de referência, compilar elementos norteadores e limitações desses instrumentos e verificar a necessidade da elaboração de um protocolo moldado à instituição.

Métodos: Estudo qualitativo realizado em um hospital público de referência. Seis fonoaudiólogos, que executam as avaliações clínicas e instrumentais da deglutição na população adulta e idosa, responderam um questionário, o qual buscou informações acerca dos protocolos utilizados na prática clínica dos participantes. Os dados obtidos foram analisados por meio do método de análise temática do conteúdo.

Resultados: Os dados foram agrupados em quatro categorias temáticas: Falta de Itens, Limitações da Oferta, Elementos Norteadores e Limitações ao Exame Instrumental. Todos os participantes afirmaram utilizar protocolos de marcação de dados em suas avaliações, referiram que os mesmos apresentam limitações e confirmaram a necessidade da criação de um protocolo de avaliação moldado às demandas da instituição.

Conclusão: Verificou-se a demanda da criação de um protocolo para avaliação clínica e instrumental da deglutição de adultos e idosos, customizado ao hospital estudado. Foram identificados elementos norteadores e limitações nos instrumentos utilizados pelos participantes, os quais contribuirão para a construção de um protocolo moldado à instituição.

Palavras-chave: Transtornos de deglutição; protocolos clínicos; avaliação; diagnóstico; indicadores

\section{ABSTRACT}

Introduction: Dysphagia is a symptom of abnormality in the deglutition process that leads to difficulty in forming or moving the food bolus safely and efficiently. Investigative methods of dysphagia are divided in clinical and instrumental assessments, which require protocols to conduct their practices. The aim of this study was to list information about protocols used by speech therapists responsible for evaluation of swallowing in adults and the elderly at a public reference hospital, to compile guidelines and limitations of these instruments and to verify the need to elaborate a protocol adapted to the institution's needs.
Clin Biomed Res. 2018;38(4):339-347

1 Programa de Pós-graduação em Medicina, Ciências Cirúrgicas, Universidade Federal do Rio Grande do Sul (UFRGS). Porto Alegre, RS, Brasil.

2 Serviço de Fonoaudiologia, Hospital de Clínicas de Porto Alegre (HCPA). Porto Alegre, RS, Brasil.

3 Departamento de Saúde e Comunicação Humana, Universidade Federal do Rio Grande do Sul (UFRGS). Porto Alegre, RS, Brasil.

Autor correspondente: Jordana Balbinot jor.balbinot@hotmail.com Faculdade de Medicina, Universidade Federal do Rio Grande do Sul (UFRGS) Rua Ramiro Barcelos, 2400. 90035-007, Porto Alegre, RS, Brasil. 
Methods: Qualitative study carried out in a public reference hospital. A total of six speech therapists who perform clinical and instrumental evaluations of swallowing in the adult and elderly population, answered a questionnaire, which sought information about the protocols used in clinical practice of participants. The obtained data were analyzed by the method of thematic content analysis.

Results: Data were grouped in four thematic categories: Lacking items, Offer limitations, Guidelines and Limitations to instrumental examination. All participants reported using data collection protocols in their evaluations, mentioned their limitations and confirmed the need for the creation of an assessment protocol to meet the institution's demands.

Conclusions: It was verified the demand for the creation of a protocol for the clinical and instrumental evaluation of swallowing in adults and the elderly, customized for the studied hospital. Guidelines and limitations in the instruments used by the participants were identified, which will contribute to the construction of a protocol adapted to the institution.

Keywords: Deglutition disorders; clinical protocols; evaluation; diagnosis; indicators

A deglutição é uma função constituída por uma série de movimentos rápidos e precisos, que conduzem alimentos e líquidos ao longo da orofaringe até o estômago, com o propósito de nutrir e hidratar o indivíduo, garantindo sua sobrevivência ${ }^{1,2}$. A disfagia é um sintoma de alteração no processo da deglutição, que acarreta dificuldade em formar ou mover o bolo alimentar de forma segura e eficiente. Esse sintoma pode estar associado a demais alterações no estado de saúde, como desnutrição, desidratação, infecções respiratórias, pneumonia aspirativa e até o óbito, e ter um forte impacto na qualidade de vida do indivíduo, ao afetar aspectos emocionais, físicos e sociais ${ }^{3,4}$.

Os métodos investigativos das alterações da deglutição se dividem em avaliação clínica e instrumental. A avaliação clínica é a base da intervenção e do acompanhamento fonoaudiológico em muitos serviços, pois possibilita a observação de sinais clínicos indicativos de disfagia de forma segura, rápida, não invasiva, reprodutível e com baixo custo ${ }^{5-7}$. Apesar disso, é subjetiva, baseia-se nos julgamentos do examinador e não dispõe de subsídios para identificar a aspiração silente ${ }^{8,9}$. Já no que tange a avaliação instrumental, a Videoendoscopia da Deglutição (VED) é um dos exames objetivos disponíveis para avaliar a deglutição. Esse exame, manejado pelo médico otorrinolaringologista, em assessoria compartilhada com a Fonoaudiologia, realiza uma avaliação global da fase faríngea da deglutição e possui grande importância para o diagnóstico da disfagia ${ }^{10}$. Ambas avaliações necessitam de instrumentos que conduzam suas práticas, para que seus resultados possam ser empregados na atuação clínica, auxiliando no diagnóstico e estabelecimento de condutas, e para fins de pesquisa.

Em um hospital público de referência, o âmbito clínico e assistencial das avaliações da deglutição de indivíduos adultos e idosos, tem evidenciado fragilidades nos protocolos empregados nessa prática. Por meio de vivências nos diferentes nichos de atuação fonoaudiológica, direcionados à disfagia, identificou-se uma expressiva necessidade de adaptação dos protocolos utilizados aos diversos contextos de avaliação, surgindo a demanda de um protocolo unificado e moldado para essa instituição.

Por conseguinte, o presente estudo objetivou levantar informações acerca dos protocolos utilizados pelos fonoaudiólogos de um hospital público de referência, que realizam avaliações clínicas e instrumentais da deglutição na população adulta e idosa, e compilar seus elementos norteadores e limitações. Além disso, buscou verificar a necessidade da elaboração de um protocolo moldado às necessidades da instituição. $O$ referido levantamento foi baseado nas demandas detectadas na rotina do hospital, a partir do ponto de vista dos profissionais da Fonoaudiologia, consultados por meio de um questionário, tendo seu embasamento teórico na literatura existente sobre o tema.

\section{MÉTODOS}

A presente pesquisa possui caráter qualitativo e foi realizada em um hospital público de referência. A partir do contato com o Serviço de Fonoaudiologia da instituição, buscou-se os profissionais da área que executam, em sua rotina assistencial, as avaliações clínicas e instrumentais da deglutição na população adulta e idosa. Foram selecionados sete fonoaudiólogos, os quais foram convidados a responder um questionário. O mesmo continha perguntas abertas e fechadas que objetivavam elencar informações acerca dos instrumentos utilizados na prática clínica dos participantes e verificar a necessidade da elaboração de um protocolo moldado às necessidades da instituição (Figura 1). Dentre os fonoaudiólogos selecionados, um não respondeu ao questionário, sendo excluído da amostra. 


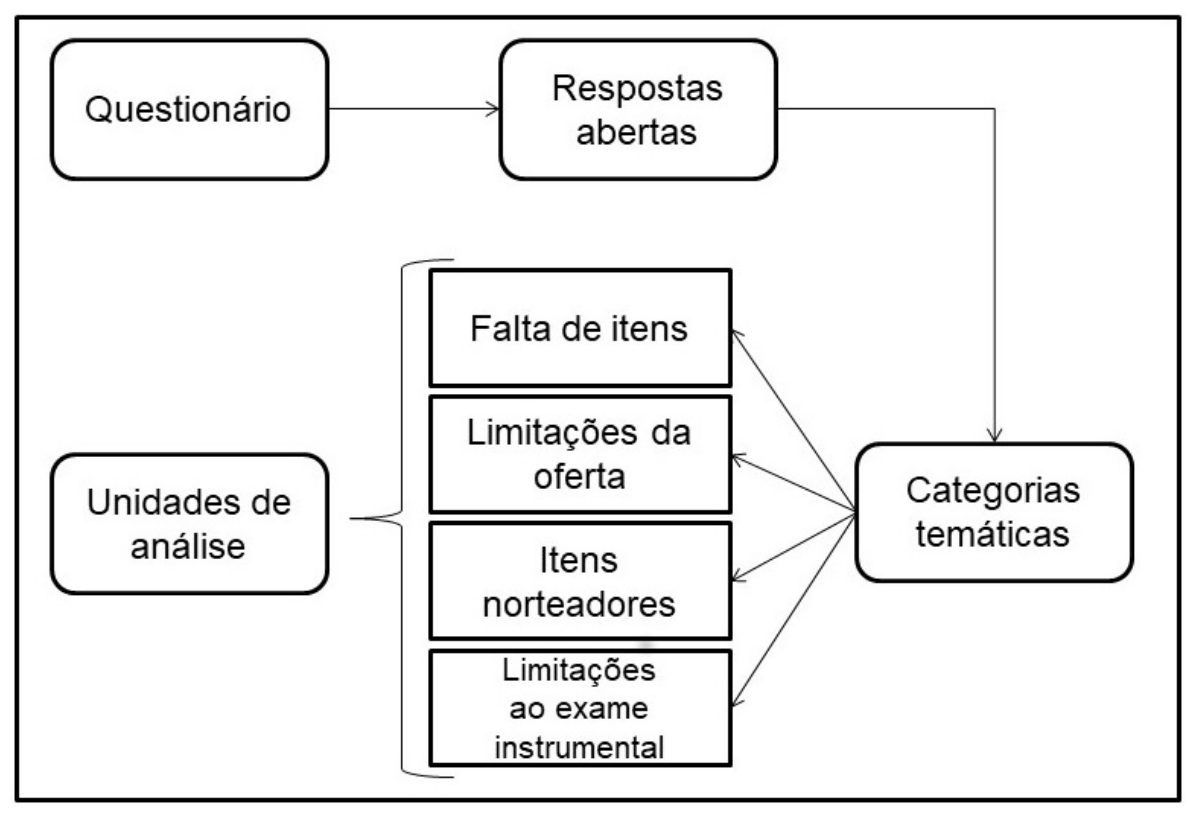

Figura 1: Esquema representativo do método de análise temática do conteúdo.

Os dados obtidos foram analisados por meio do método de análise temática do conteúdo, o qual visa realizar uma descrição do assunto de maneira objetiva e sistemática ${ }^{11}$. Além disso, busca obter indicadores de descrição do conteúdo que permitam a inferência de conhecimentos relativos ao objeto estudado ${ }^{12}$. A análise temática, que é um tipo de análise do conteúdo, está ligada a uma afirmação a respeito de determinado assunto, sobre o qual, a presença ou frequência de um determinado dado traz significado para o objetivo investigado ${ }^{11}$. Por meio deste método, a partir do contexto das respostas dos participantes e de acordo com os objetivos da pesquisa, os dados do presente estudo foram categorizados em temas, Falta de Itens, Limitações da Oferta, Elementos Norteadores e Limitações ao Exame Instrumental. Em cada tema foram agrupadas as unidades de análise das categorias por frequência, ou seja, por repetição de conteúdos comuns à maioria dos respondentes e por relevância implícita, quando a unidade tinha relevância para o estudo, mas não se repete no relato de outros participantes ${ }^{11}$. A Figura 1 retrata um esquema representativo do método utilizado.

Este estudo foi submetido ao Comitê de Ética em Pesquisa (CEP) da instituição, aprovado sob o número 17-0532 e conduzido conforme a resolução 466/12 do Conselho Nacional de Saúde.

\section{RESULTADOS}

Os dados foram agrupados em quatro categorias temáticas, de acordo com os norteadores e limitações obtidos mediante o questionário: Falta de Itens,
Limitações da Oferta, Elementos Norteadores e Limitações ao Exame Instrumental. Tais categorias e suas respectivas unidades de análise serão descritas posteriormente. Quanto aos tipos de avaliações realizadas, seis participantes informaram realizar avaliações clínicas, e dois, avaliações instrumentais da deglutição (por meio da VED). Todos os fonoaudiólogos afirmaram utilizar protocolos de marcação de dados em suas avaliações, bem como referiram que os mesmos apresentam limitações, confirmando que percebem a necessidade da criação de um protocolo de avaliação moldado às demandas da instituição.

Os participantes elencaram algumas limitações gerais acerca dos protocolos que usam em sua prática clínica. Dentre elas, que os instrumentos são demasiado extensos para a realidade assistencial, porém incompletos, sendo necessária a utilização de mais de um protocolo para obtenção das informações ou emprego de notas complementares para registro dos acontecimentos. Houve grande heterogeneidade entre os instrumentos aplicados por cada fonoaudiólogo, sendo utilizados até quatro protocolos diferentes por um mesmo profissional perante a avaliação clínica da deglutição. Além disso, os participantes referiram que a maioria dos instrumentos disponíveis são traduzidos, porém não são validados para a população brasileira. Outra limitação citada refere-se à dificuldade na determinação do nível de gravidade da disfagia, pois as escalas de classificação vigentes contêm muitos detalhes, não contemplando a realidade dos achados clínicos. 
As Tabelas 1 e 2 contêm as unidades de análise das categorias Falta de Itens e Limitações da Oferta. Nelas foram elencados os itens identificados pelos fonoaudiólogos, os quais não são contemplados nos protocolos utilizados em suas rotinas, suas descrições e a frequência de participantes que referiram cada limitação.

$\mathrm{Na}$ Tabela 3 encontram-se os elementos norteadores elencados pelos fonoaudiólogos, contidos nos protocolos usualmente empregados em suas rotinas clínicas.

Além das considerações já apontadas, na categoria Limitações ao Exame Instrumental, os dois participantes que afirmaram realizar este tipo de avaliação, referiram a necessidade de implementar alguns indicadores não contemplados nos protocolos de avaliação do exame de VED. Tais indicadores contemplam a análise do mecanismo velofaríngeo, a efetividade da coaptação glótica, a presença, quantidade e localização de estase prévia e após deglutições e a avaliação da sensibilidade laríngea.

\section{DISCUSSÃO}

Os norteadores e limitações, elencados pelos fonoaudiólogos da amostra, vão ao encontro dos achados da literatura, no que tange os indicadores essenciais que devem ser analisados durante as avaliações da deglutição e serão discutidos a seguir conforme as categorias e unidades de análise explanadas.

Para além de determinar as alterações e a gravidade da disfagia, a avaliação da deglutição busca, principalmente, direcionar as condutas clínicas e a reabilitação fonoaudiológica. Portanto, perante a avaliação deve-se identificar as fases da deglutição comprometidas e testar a efetividade de estratégias que possam minimizar os sinais e sintomas de disfagia do indivíduo avaliado ${ }^{13,14}$. Ambos aspectos foram citados como norteadores da avaliação clínica da deglutição por 3 dos entrevistados (Tabela 3 ).

Similarmente, o quadro clínico e o histórico de saúde, que incluem informações sobre a doença de base, complicações médicas, peso, estado nutricional,

Tabela 1: Falta de itens nos protocolos utilizados.

\begin{tabular}{ccc}
\hline Falta de Itens & Descrição & Frequência \\
\hline Histórico clínico & Doença de base e complicações & 1 \\
Nível Cognitivo & Nível de consciência e estado de alerta & 2 \\
Fadiga & Durante a alimentação & 1 \\
Anatomofisiologia & Tônus, sensibilidade e mobilidade & 2 \\
Estase/resíduo & Presente em cavidade oral & 1 \\
Fase(s) da deglutição & Na qual ocorre alteração & 1 \\
\hline
\end{tabular}

Fonte: Elaborado pelo autor.

Tabela 2: Limitações dos protocolos acerca da oferta.

\begin{tabular}{ccc}
\hline Limitações da Oferta & Descrição & Frequência \\
\hline Consistência sólida & Não consta nos protocolos & 2 \\
Demanda livre & Não consta nos protocolos & 2 \\
Deglutição de saliva & Não consta nos protocolos & 1 \\
Tipo de utensílio & Colher, seringa, copo & 1 \\
Volumes & Apenas teste de pequenos volumes & 1 \\
\hline
\end{tabular}

Fonte: Elaborado pelo autor.

Tabela 3: Elementos norteadores nos protocolos utilizados.

\begin{tabular}{cc}
\hline Elementos Norteadores & Frequência \\
\hline Tempo de trânsito oral & 1 \\
Excursão hiolaríngea & 1 \\
Presença de deglutições múltiplas & 1 \\
Sinais sugestivos de penetração/aspiração & 4 \\
Qualidade vocal & 1 \\
Utilização e efetividade de manobras & 3 \\
Espaço para definição de condutas & 3
\end{tabular}

Fonte: Elaborado pelo autor. 
cognitivo, metabólico e cardíaco, são demais aspectos decisivos para direcionar o prognóstico e a conduta fonoaudiológica. Consequentemente, é primordial investigá-los durante a avaliação do paciente disfágico ${ }^{15}$. Apesar de sua importância perante a avaliação, de acordo com os fonoaudiólogos da amostra, tais itens não são contemplados nos protocolos empregados em sua rotina clínica.

Ainda, como apontado pelos entrevistados, o nível cognitivo deve ser examinado atentamente, antes da oferta alimentar, pois para a segurança da deglutição o paciente deve estar alerta e ser capaz de receber alimentos por via oral ${ }^{5}$. O nível de consciência rebaixado interfere na colaboração do paciente, na prontidão das respostas reflexas e na proteção das vias aéreas, desfavorecendo a deglutição eficiente e aumentando o risco de pneumonia aspirativa ${ }^{16,17}$.

Diversas doenças de base, que causam disfagia, afetam a execução da mastigação e da deglutição pela diminuição do tônus e da sensibilidade das regiões oral e faríngea, bem como pela incoordenação dos movimentos necessários para a execução dessas funções. Por conseguinte, a avaliação da anatomia e da neurofisiologia da dinâmica da deglutição é fundamental, pois, se alterada, pode gerar mudanças no direcionamento do alimento, trazendo risco ao indivíduo ${ }^{16,18}$. Contudo, 2 fonoaudiólogos referiram que a avaliação da anatomofisiologia não consta nos instrumentos vigentes (Tabela 1).

A presença de estase ou resíduo em cavidade oral também é um elemento que deve receber a atenção do avaliador, pois é um indicativo que as fases preparatória-oral e/ou oral da deglutição encontram-se ineficientes, pela dificuldade em controlar e propulsionar o alimento em direção à faringe ou por uma redução da sensibilidade intra-oral ${ }^{13,15,19}$. Isso posto, o tônus, a sensibilidade, a mobilidade e a integridade das estruturas, bem como a preservação das funções e a presença de resíduo em cavidade oral devem ser observados, já que são fundamentais no diagnóstico e no direcionamento da reabilitação ${ }^{14}$.

No que tange a decisão do clínico acerca do teste de diferentes consistências e volumes, o nível de consciência, as condições clínicas gerais e as consistências que o paciente consome por via oral, em sua rotina, devem ser levadas em conta, visto que as características do bolo alimentar interferem diretamente na dinâmica da deglutição ${ }^{20}$.

Quando comparada à deglutição de alimentos menos consistentes, a consistência sólida exige uma maior manipulação oral ${ }^{21}$, podendo ocasionar lentidão no trânsito oral e faríngeo e aumento no risco de penetração e aspiração ${ }^{20}$. O tempo de trânsito oral é caracterizado como o período entre a captação completa do bolo alimentar até o disparo do reflexo da deglutição. Esse, quando aumentado, indica que a fase preparatória oral está inadequada, evidenciando dificuldade na organização e propulsão do alimento e atraso no disparo do reflexo da deglutição ${ }^{15,19}$.

Já a consistência líquida, por seu difícil controle em cavidade oral, pode ocasionar escape posterior e aumento no risco de aspiração durante a avaliação ${ }^{22,23}$. Em contrapartida, os alimentos pastosos, por sua propriedade coesa, são mais facilmente controlados na cavidade oral, sendo considerada, na maioria dos casos, a consistência mais segura e usualmente indicada para iniciar a avaliação da deglutição ${ }^{13,20,22}$.

Sempre que possível, a avaliação deve abarcar as consistências líquida, pastosa e sólida, em diversos volumes, de acordo com os objetivos do exame e o estado do paciente. As ofertas devem iniciar de maneira controlada, com volumes menores ${ }^{15}$, porém, de acordo com o andamento da avaliação, pode-se utilizar a "livre demanda", a qual torna mais fidedigno o olhar sobre o gerenciamento da alimentação e o manejo da deglutição do indivíduo. Apesar da importância desses parâmetros para a avaliação da deglutição, conforme o relato de 2 fonoaudiólogos da amostra (Tabela 2), o teste da livre demanda e da consistência sólida não são contemplados nos protocolos utilizados.

Outra característica importante acerca da oferta é o tipo de utensílio utilizado, que pode ser a colher e a seringa para volumes controlados, ou o copo para volumes maiores ${ }^{13}$. Vale ressaltar que, sempre que possível, a avaliação deve simular uma refeição próxima à habitual do paciente, quanto aos tipos de alimentos, consistências, volumes, utensílios e temperatura. Nesse sentido, é essencial a identificação do utensílio empregado rotineiramente, com vistas a utilizá-lo na avaliação, ou recomendar sua suspensão caso seja inadequado.

Nas ocasiões em que o paciente apresenta disfagia e/ou aspiração conhecidas, a deglutição de saliva pode ser um bom parâmetro de avaliação, contudo, foi sinalizado por 1 dos entrevistados (Tabela 2) que este aspecto não consta nos instrumentos empregados. O teste de deglutição de saliva fornece informações anatômicas profundas e superficiais, sendo fisiológica, segura e viável ${ }^{24}$.

Dois importantes norteadores nas avaliações, identificáveis por meio da ausculta cervical, por monitoramento visual e digital, são a excursão do complexo hiolaríngeo e o número de deglutições ${ }^{19}$. A primeira é fundamental para que ocorra o direcionamento adequado do bolo alimentar e a proteção das vias aéreas ${ }^{15,19}$ e o segundo indica a quantidade de deglutições necessárias para a completa ingestão do bolo ofertado ${ }^{9}$. Duas deglutições são consideradas normais em indivíduos com 
ou sem disfagia, já a presença de deglutições múltiplas ocorre quando são necessárias três ou mais deglutições para a limpeza do trato vocal em até um minuto após a oferta ${ }^{9}$. Dificuldades nesses aspectos podem acarretar risco de penetração e aspiração, podendo indicar dificuldade de propulsão do alimento, alteração do reflexo da deglutição, comprometimento da transição entre a fase oral para a faríngea da deglutição e presença de estase nas estruturas orais e faríngeas ${ }^{15,19}$.

Os sinais clínicos indicativos de penetração e aspiração são a tosse, o engasgo e o pigarro e possuem suma importância na avaliação da deglutição, como verificou-se no presente estudo, no qual 4 dos fonoaudiólogos entrevistados citaram tais elementos como norteadores presente nos protocolos utilizados (Tabela 3). Os sinais clínicos investigam o funcionamento dos mecanismos de proteção das vias aéreas e a segurança durante a alimentação por via oral, podendo indicar penetração ou aspiração laringotraqueal, a presença de resíduos alimentares e sensibilidade na região laríngea ${ }^{15}$.

A qualidade vocal também deve receber a atenção do avaliador e é um norteador geralmente encontrado nos protocolos de avaliação, como sinalizado por 1 dos entrevistados deste estudo (Tabela 3). A presença de voz molhada após a deglutição é um sinal sugestivo da presença de material ofertado na laringe, indicando risco de penetração/aspiração ${ }^{1,25}$.

Vale ressaltar que a ausência de sinais clínicos não significa inexistência de penetração ou aspiração. Essas condições representam os casos de aspiração silente, nos quais ocorre aspiração antes, durante ou após a deglutição, sem sinais clínicos sugestivos ${ }^{5}$. Sabe-se que, apesar da avaliação clínica da deglutição ser considerada sensível para detectar e classificar as alterações das fases preparatória oral, oral e faríngea da deglutição ${ }^{7}$, ela não é capaz de identificar cerca de $50 \%$ dos pacientes que tem risco de aspiração ${ }^{9}$.

Em suspeita de aspiração silente, uma avaliação complementar instrumental pode ser indicada. Contudo, sabe-se que a utilização de procedimentos objetivos de diagnóstico nem sempre é acessível na prática diária, devido a restrições econômicas, limitação de profissionais e falta de equipamentos ${ }^{5,6}$. É por essa razão que os resultados de uma avaliação clínica confiável são tão importantes. Por meio deles pode-se predizer a necessidade de uma avaliação instrumental, o que otimiza tempo e economiza recursos financeiros, sem comprometer o cuidado ao paciente ${ }^{19}$. Ademais, a avaliação clínica prévia ao exame instrumental fornece informações que nortearão o exame objetivo, como consistências, volumes e manobras que devem ser testados, permitindo ao fonoaudiólogo saber o que procura ao indicar o exame ideal ${ }^{26}$.

Os dados obtidos por uma avaliação clínica, somados aos de uma avaliação instrumental, que examine de forma objetiva a fase faríngea, tal como a VED, propiciam um diagnóstico mais completo e preciso da função deglutitória? .

Todos os indicadores anteriormente citados são importantes e devem ser observados durante as avaliações clínicas e objetivas da deglutição. Entretanto, a avaliação instrumental permite a análise de outros aspectos obtidos pelas imagens objetivas.

As etapas oral e faríngea da deglutição são compostas por uma série de eventos neuromusculares rapidamente executados: transporte oral do bolo, vedamento velofaríngeo, fechamento e elevação laríngea, abertura cricofaríngea e peristaltismo ${ }^{27}$. Algumas dessas características fisiológicas podem ser avaliadas durante a VED e foram relatadas pelos participantes desse estudo como não referidas nos protocolos utilizados.

O processo de deglutição envolve o "jogo pressórico" dos esfíncteres labial, velofaríngeo, laríngeo e esofágico, que se alterado aumenta o risco de aspiração. A oclusão adequada do esfíncter velofaríngeo auxilia na manutenção das forças de propulsão e possibilita a adequada pressão intra-oral para o direcionamento do alimento ao esôfago, evitando seu escape pela cavidade nasal ${ }^{15,28}$. Esta função pode ser testada por meio da VED com o posicionamento da fibra óptica na região do esfíncter velofaríngeo e solicitação de movimentos que induzam seu funcionamento, porém, de acordo com os 2 entrevistados que realizam essa avaliação objetiva, tal elemento não é abarcado nos protocolos utilizados.

A coaptação glótica também pode ser avaliada durante a VED e configura-se pelo fechamento automático e completo da glote durante a deglutição, sendo considerada a mais importante forma de proteção das vias aéreas ${ }^{28}$.

Durante o exame instrumental é muito comum a ocorrência de estase, ou resíduo faríngeo, que é definida como a retenção do todo ou partes do material ofertado nas regiões de valécula e seios piriformes, após duas deglutições. Essa alteração pode ser causada por movimentação ou força da musculatura reduzidas e/ou por diminuição da sensibilidade do trato vocal. É aceitável a presença de estase de $10 \%$ a $15 \%$ da oferta após a primeira deglutição 9,29 . Nesse estudo, os 2 fonoaudiólogos que realizam avaliações objetivas por meio da VED em sua prática clínica, referiram que a estase não consta nos instrumentos empregados em sua rotina. Porém, na literatura encontra-se a escala 
BRACS - Boston Residue and Clearance Scale, instrumento que foi desenvolvido com o intuito de avaliar a presença, a quantidade e a localização de resíduo faríngeo, bem como verificar a eficiência do clareamento da estase ${ }^{29,30}$ :

A capacidade do paciente em realizar uma deglutição espontânea em resposta ao resíduo faríngeo é imprescindível, pois a diminuição de sensibilidade aumenta o risco de penetração e aspiração ${ }^{29}$. Nesse contexto, a informação acerca da sensibilidade faz-se fundamental, principalmente quando há suspeita de aspiração silente. $O$ teste de sensibilidade pode ser realizado por meio do toque com a extremidade distal do fibroscópio nas estruturas do trato vocal. Apesar do toque do equipamento representar um estímulo maior do que aquele gerado pelo alimento, pacientes insensíveis ao mesmo podem apresentar redução ou ausência de sensibilidade durante as refeições ${ }^{31}$. Contudo, os 2 entrevistados referiram que o teste de sensibilidade também não é contemplado nos protocolos utilizados.

Segundo Pilz e colaboradores (2016), enquanto a popularidade da VED está aumentando, pesquisas de padronização e validação dos critérios de medidas desses exames ficaram para trás, visto que a literatura raramente descreve os protocolos ou as variáveis de análise em suficiente detalhe ${ }^{10}$.

Existe uma grande diversidade de instrumentos descritos na literatura, porém, não há um protocolo padrão universalmente aceito para avaliação clínica e instrumental da deglutição, ou um consenso sobre a utilização de um único instrumento ${ }^{22}$. Esses precisam ser moldados aos diferentes ambientes, às equipes e à dinâmica da instituição na qual são empregados. Cabe ao profissional elencar o instrumento que se adapte à sua realidade de atuação, ao funcionamento da instituição e ao perfil dos pacientes que serão avaliados $^{32}$. A utilização de protocolos permeia a atuação responsável e comprovada, permite atuar de forma planejada, documentando procedimentos e avaliando padrões de trabalho ${ }^{33,34}$.
No presente estudo verificou-se que os fonoaudiólogos, em sua rotina clínica, fazem adaptações nos protocolos que utilizam durante as avaliações da deglutição e que os mesmos não atendem integralmente às necessidades assistenciais das equipes. Além disso, que os instrumentos são demasiado extensos para a realidade assistencial, porém incompletos, e que há dificuldade na determinação do nível de gravidade da disfagia.

Por meio do questionário, respondido pelos fonoaudiólogos, foi possível identificar os norteadores e as limitações dos protocolos utilizados em sua prática clínica. Tais norteadores consistem na avaliação do tempo de trânsito oral, da excursão hiolaríngea, do número de deglutições, dos sinais clínicos de penetração/aspiração, da qualidade vocal, bem como o teste de manobras e o espaço para definição de condutas. Quanto às limitações, foi possível identificar a falta de elementos essenciais para avaliação, como o histórico clínico, o nível cognitivo, a fadiga durante a alimentação, a avaliação da anatomofisiologia, a presença de estase em cavidade oral e a demarcação da fase da deglutição alterada. Verificou-se ainda a falta de itens referentes à oferta, devido à não serem contemplados elementos como o tipo de utensílio e o volume a ser testado, o teste da consistência sólida, de livre demanda e de deglutição de saliva. Perante o exame instrumental, por meio da VED, foi identificado que os protocolos não abarcam a análise do mecanismo velofaríngeo, a efetividade da coaptação glótica e a avaliação das características da estase e da sensibilidade laríngea.

Ademais, o presente estudo possibilitou identificar a demanda da criação de um protocolo para avaliação clínica e instrumental da deglutição, de adultos e idosos, customizado ao hospital estudado.

\section{Conflitos de Interesse}

Os autores declaram não ter conflitos de interesse.

\section{REFERÊNCIAS}

1. McCullough $\mathrm{GH}$, Mccullough $\mathrm{KC}$ Clinical evaluation of swallowing function for institutionalized elderly: balancing the scales for safety and quality of life. Journal of Legal Nurse Consulting. 2014;25(2):12-7.

2. Oliveira T, Braz KCC. Desnutrição e desidratação. In: Jotz GP, Angelis EC, Barros APB, editores. Tratado da deglutição e disfagia no adulto e na criança. Rio de Janeiro: Revinter; 2009. p. $292-6$
3. Clavé P, Shaker R. Dysphagia: current reality and scope of the problem. Nat Rev Gastroenterol Hepatol. 2015;12(5):259-70. http:// dx.doi.org/10.1038/nrgastro.2015.49. PMid:25850008.

4. Portas J, Guedes RLV. Qualidade de vida e deglutição: como avaliar na prática clínica. In: Marchezan IQ, Silva HJ, Tomé MC, editores. Tratado das especialidades em Fonoaudiologia. São Paulo: Guanabara Koogan; 2014. p. 21-4
5. Hammond CAS, Goldstein LB. Cough and aspiration of food and liquids due to oral-pharyngeal dysphagia: ACCP evidence-based clinical practice guidelines. Chest. 2006;129(1 Suppl):154S-68S. http://dx.doi. org/10.1378/chest.129.1_suppl.154S. PMid:16428705.

6. Padovani AR, Moraes DP, Sassi FC, Andrade CR. Avaliação clínica da deglutição em unidade de terapia intensiva. CoDAS. 2013;25(1):1- 
7. http://dx.doi.org/10.1590/ S2317-17822013000100002. PMid:24408163.

7. Santoro PP, Furia CLB, Forte AP, Lemos EM, Garcia RI, Tavares RA, et al. Avaliação otorrinolaringológica e fonoaudiológica na abordagem da disfagia orofaríngea: proposta de protocolo conjunto. Rev Bras Otorrinolaringol. 2011;77(2):201-13.

8. Leder SB, Espinosa JF. Aspiration risk after acute stroke: comparison of clinical examination and fiberoptic endoscopic evaluation of swallowing. Dysphagia. 2002;17(3):214-8. http:// dx.doi.org/10.1007/s00455-002-00547. PMid:12140648.

9. Park WY, Lee TH, Ham NS, Park JW, Lee YG, Cho SJ, et al. Adding endoscopist-directed flexible endoscopic evaluation of swallowing to the videofluoroscopic swallowing study increased the detection rates of penetration, aspiration and pharyngeal residue. Gut Liver. 2015;9(5):623-8. http://dx.doi.org/10.5009/gnl14147. PMid:25473074.

10. Pilz W, Vanbelle S, Kremer B, van Hooren MR, van Becelaere T, Roodenburg N, et al. Observers' agreement on measurements in fiberoptic endoscopic evaluation of swallowing. Dysphagia. 2016;31(2):180-7. http://dx.doi. org/10.1007/s00455-015-9673-7. PMid:26803774.

11. Campos CJG. Método de análise de conteúdo: ferramenta para a análise de dados qualitativos no campo da saúde. Rev Bras Enferm. 2004;57(5):6114. http://dx.doi.org/10.1590/ S0034-71672004000500019. PMid:15997809.

12. Gerhardt TE, Ramos ICA, Riquinho DL, Santos DL. Estruturo do projeto de pesquisa. In: Gerhardt TE, Silveira DT, editores. Métodos de pesquisa. Porto Alegre: Editora UFRGS; 2009. p. 65-88.

13. Vale-Prodomo LP, Angelis EC, Barros APB. Avaliação clínica fonoaudiológica das disfagias. In: Jotz GP, Angelis EC, Barros APB, editores. Tratado da deglutição e disfagia no adulto e na criança. Rio de Janeiro: Revinter; 2009. p. 61-7.

14. Netto IP, Angelis EC, Barros APB. Princípios da reabilitação das disfagias orofaríngeas. In: Jotz GP,
Angelis EC, Barros APB, editores. Tratado da deglutição e disfagia no adulto e na criança. Rio de Janeiro: Revinter; 2009. p. 330-41.

15. Silva RG. Disfagia neurogênica em adultos: uma proposta para avaliação clínica. In: Furkim AM, Santini, CS, editores. Disfagias orofaríngeas. Carapicuiba: Pró-Fono; 1999. p. 3547.

16. Abdulmassih EMS, Macedo ED Fo, Santos RS, Jurkiewicz AL. Evolução de pacientes com disfagia orofaríngea em ambiente hospitalar. Arq Int Otorrinolaringol. 2009;13(1):55-62.

17. Hu X, Lee JS, Pianosi PT, Ryu $\mathrm{JH}$. Aspiration-related pulmonary syndromes. Chest. 2015;147(3):81523. http://dx.doi.org/10.1378/ chest.14-1049. PMid:25732447.

18. Tavares TE, Carvalho CMRG. Características de mastigação e deglutição na Doença de Alzheimer. Rev CEFAC. 2012;14(1):122-37. http://dx.doi.org/10.1590/S151618462011005000123.

19. Padovani AR. Protocolo fonoaudiológico de introdução e transição da alimentação por via oral para pacientes com risco para disfagia (PITA) [dissertação]. São Paulo: Universidade de São Paulo; 2010. http://dx.doi.org/10.11606/D.5.2010. tde-19042010-110604.

20. Santana L, Fernandes A, Brasileiro AG, Abreu AC. Critérios para avaliação clínica fonoaudiológica do paciente traqueostomizado no leito hospitalar e internamento domiciliar. Rev CEFAC. 2014;16(2):524-36. http://dx.doi.org/10.1590/1982021620144712.

21. Carneiro D, Belo LR, Coriolano MGWS, Asano AGC, Lins OG. Qualidade de vida em disfagia na Doença de Parkinson: uma revisão sistemática. Rev CEFAC. 2013;15(5):1347-56. http:// dx.doi.org/10.1590/S151618462013005000050.

22. Leder SB, Suiter DM, Warner HL. Answering orientation questions and following single-step verbal commands: effect on aspiration status. Dysphagia. 2009;24(3):290-5. http:// dx.doi.org/10.1007/s00455-0089204-x. PMid:19263106.

23. Kuhlemeier KV, Palmer JB, Rosenberg D. Effect of liquid bolus consistency and delivery method on aspiration and pharyngeal retention in dysphagia patients. Dysphagia. 2001;16(2):119-22. http://dx.doi. org/10.1007/s004550011003. PMid:11305221.

24. Hartl DM, Kolb F, Bretagne E, Marandas P, Sigal R. Cine Magnetic Resonance Imaging with singleshot fast spin echo for evaluation of dysphagia and aspiration. Dysphagia. 2006;21(3):156-62. http://dx.doi. org/10.1007/s00455-006-9026-7. PMid:16718621.

25. Groves-Wright KJ, Boyce S, Kelchner L. Perception of wet vocal quality in identifying penetration/aspiration during swallowing. J Speech Lang Hear Res. 2010;53(3):620-32. http://dx.doi.org/10.1044/10924388(2009/08-0246). PMid:20029051.

26. Netto IP, Angelis EC, Barros APB. Correlação das avaliações clínica, videofluoroscópica e nasofribolaringoscópica e sua implicação na reabilitação. In: Jotz GP, Angelis EC, Barros APB, editores. Tratado da deglutição e disfagia no adulto e na criança. Rio de Janeiro: Revinter; 2009. p. 348-51.

27. Logemann JA, Kahrilas PJ. Relearning to swallow after strokeapplication of maneuvers and indirect biofeedback: a case study. Neurology. 1990;40(7):1136-8. http:// dx.doi.org/10.1212/WNL.40.7.1136. PMid:2356016.

28. Marchesan IQ. Deglutiçãonormalidade. In: Furkim AM, Santini CS, editores. Disfagias orofaríngeas. Carapicuiba: Pró-Fono; 1999. p. 3-18.

29. Kaneoka AS, Langmore SE, Krisciunas GP, Field K, Scheel R, McNally E, et al. The Boston Residue and Clearance Scale: preliminary reliability and validity testing. Folia Phoniatr Logop. 2013;65(6):312-7. http://dx.doi.org/10.1159/000365006. PMid:25033761.

30. Ardenghi LG. Influência da dupla tarefa na deglutição de pacientes portadores da Doença de Parkinson [tese]. Porto Alegre: Universidade Federal do Rio Grande do Sul; 2016.

31. Langmore SE, Kenneth SMA, Olsen N. Fiberoptic endoscopic examination of swallowing safety: a new procedure. Dysphagia. 1988;2(4):216-9. http:// dx.doi.org/10.1007/BF02414429. PMid:3251697. 
Protocolos de avaliação da deglutição

32. Etges CL, Scheeren B, Gomes E, Barbosa LDR. Instrumentos de rastreio em disfagia: uma revisão sistemática. CoDAS. 2014;26(5):343-9. http:// dx.doi.org/10.1590/23171782/20142014057. PMid:25388065.
33. Padovani AR, Moraes DP, Mangili LD, Andrade CRF. Protocolo fonoaudiológico de avaliação do risco para disfagia (PARD). Rev Soc Bras Fonoaudiol. 2007:12(3):199-205. http://dx.doi.org/10.1590/S151680342007000300007.
34. Pimentel PCV. Proposta de elaboração de um protocolo de avaliação fonoaudiológica da disfagia infantil [trabalho de conclusão de curso]. Belo Horizonte: Universidade Federal de Minas Gerais; 2009.

Recebido: 30 abr, 2018 Aceito: 14 jan, 2019 